\title{
Mindfulness disposicional, espiritualidad y religión y su papel como factores protectores del consumo de sustancias en adolescentes mexicanos Dispositional mindfulness, spirituality and religion, and their role as protective factors of substance use in Mexican adolescents
}

Axel Ramírez-Garduño ${ }^{1}$ Marcela Veytia López² ${ }^{2}$ Rosalinda Guadarrama Guadarrama² y Jennifer Lira Mandujano ${ }^{3}$

Palabras clave: mindfulness; espiritualidad; religión; consumo de sustancias; drogas; adolescentes mexicanos; salud; alcohol; tabaco; adicciones Keywords: mindfulness; spirituality; religion; substance use; drugs; Mexican teenagers; health; alcohol; tobacco; addictions

Recibido en: 30-03-2020 / Aceptado en: 24-06-2020

\section{Resumen}

Introducción: Los adolescentes son particularmente vulnerables a conductas de riesgo, como el consumo de sustancias psicoactivas (alcohol, tabaco y drogas ilegales), lo que puede conducir a otros trastornos a lo largo de la vida. A medida que el consumo de sustancias ha aumentado a través de los años, es importante la integración de nuevas perspectivas en la cultura de la prevención y el cuidado en la juventud y la población general. Este estudio tuvo como objetivo evaluar las relaciones entre mindfulness disposicional, espiritualidad y religión, y su papel como factores protectores del consumo de sustancias psicoactivas en adolescentes, además de analizar las diferencias en las puntuaciones de mindfulness y espiritualidad entre consumidores y no consumidores de sustancias, y entre los diferentes grupos religiosos/espirituales.

Método: Se llevó a cabo un estudio transversal, donde se evaluó a 433 adolescentes estudiantes de nivel medio superior de entre 14 y 19 años (Media $=16.27 \pm .91$ ). Se ejecutaron pruebas de correlación estadística para evaluar la relación entre las variables, además de las pruebas $t$ de Student y ANOVA unidireccional para explorar las diferencias en las puntuaciones de mindfulness y espiritualidad entre los grupos de consumidores y no consumidores de sustancias, y entre los grupos religiosos/espirituales, respectivamente. Finalmente, por medio de un análisis de regresión logística binaria, se evaluó el grado de predicción de consumo de sustancias psicoactivas.

Resultados: La muestra reportó mayor consumo de sustancias psicoactivas a comparación de la última encuesta nacional. El mindfulness disposicional se correlacionó de manera positiva con la espiritualidad y la religión, y de manera inversa con el consumo de sustancias, con una fuerza de ${ }^{1}$ Universidad Autónoma del Estado de México, Facultad de Medicina. E-mail: psic.axelrmz@hotmail.com

${ }^{2}$ Universidad Autónoma del Estado de México, Instituto de Estudios Sobre la Universidad

${ }^{3}$ Universidad Nacional Autónoma de México, FES Iztacala

(C) Universidad De La Salle Bajío (México) 
leve a moderada. Además, los individuos que consumían sustancias psicoactivas tenían puntuaciones significativamente menores en mindfulness a quienes nunca habían consumido en absoluto, pero no se encontraron diferencias con respecto a las puntuaciones de espiritualidad. Las puntuaciones en mindfulness y espiritualidad fueron significativamente mayores en quienes pertenecían formalmente a una religión. Finalmente, la pertenencia a una religión, seguida del mindfulness disposicional fueron las mejores variables predictoras como factor de protección de consumo de alcohol y drogas, mientras que la espiritualidad no fue una variable que contribuía significativamente a los modelos y por lo tanto fue eliminada.

Discusión o Conclusión: El mindfulness disposicional, la espiritualidad y la religión son variables relacionadas entre sí. La pertenencia a la religión y el mindfulness disposicional podrían ser un mejores factor protector ante el consumo de sustancias en adolescentes que la espiritualidad. El mindfulness disposicional, además, fue mayor en quienes practicaban formalmente una religión. Finamente, el consumo de sustancias psicoactivas podría alterar las habilidades relacionadas con el mindfulness, pero es necesaria más investigación que pueda complementar y esclarecer estos resultados en población mexicana.

\begin{abstract}
Introduction: Adolescents are particularly vulnerable to risky behaviors, such as the use of psychoactive substances (alcohol, tobacco, and illegal drugs), which can lead to other disorders throughout life. As substance use has risen over the years, the integration of new perspectives into the culture of prevention and care in youth and the general population is important. This study aimed to evaluate the relationships between dispositional mindfulness, spirituality and religion, and their role as protective factors of the psychoactive substance use in adolescents, analyzing additionally the differences in mindfulness and spirituality scores between substance and nonsubstance users, and between the different religious/spiritual groups.

Method: A cross-sectional study was carried out, in which 433 adolescent high school students between the ages of 14 and 19 were evaluated (Mean $=16.27 \pm .91)$. Statistical correlation tests were performed to assess the relationship between variables, in addition to Student t-test and oneway ANOVA to explore differences in mindfulness and spirituality scores of substance and nonsubstance users, and between religious/spiritual groups, respectively. Finally, by means of a binary logistic regression analysis, the degree of prediction of psychoactive substance use was evaluated.
\end{abstract}


Results: The sample reported higher consumption of psychoactive substances compared to the last national survey. Dispositional mindfulness was positively correlated with spirituality and religion, and inversely correlated with substance use, with mild to moderate strength. Additionally, individuals who used psychoactive substances had significantly lower mindfulness scores than those who had never used it at all, but no differences were found regarding spirituality scores. The scores in mindfulness and spirituality were significantly higher in those who formally belonged to a religion. Finally, belonging to a religion, followed by dispositional mindfulness were the best predictor variables as a protection factor for alcohol and drug consumption, while spirituality was not a variable that significantly contributed to the models and was therefore eliminated.

Discussion or Conclusion: Dispositional mindfulness, spirituality, and religion are interrelated variables. Belonging to religion and dispositional mindfulness may be a better protective factor against substance use in adolescents than spirituality. Dispositional mindfulness was also greater in those who formally belonged to a religion. Finally, the psychoactive substance use could alter mindfulness-related abilities, but more research is needed to complement and clarify these results in the Mexican population.

\section{Introducción}

La investigación en psicología de la salud se ha encargado de estudiar el papel de comportamientos individuales y colectivos que fomenten el bienestar de las personas en sociedad, de manera que puedan ayudar a prevenir síntomas de psicopatología como la ansiedad, la depresión y el abuso de sustancias psicoactivas. En las últimas tres décadas, diversas investigaciones denotan un creciente interés en el estudio del mindfulness, la espiritualidad, y la religión como aspectos funcionales del individuo en términos de resiliencia, factores protectores de la salud y afrontamiento (Carmody, Reed, Kristeller y Merriam, 2008; Koenig, 2012; Tabei, Zarei, y Joulaei, 2016; Zinnbauer et al., 1997).

El mindfulness, cuya traducción al español más cercana, pero menos precisa es "atención plena", tiene sus orígenes fundamentales en la meditación de la tradición budista, y dentro del argot científico ha sido definido como "prestar atención de una manera particular: de manera deliberada, en el momento presente y sin emitir juicios" (Kabat-Zinn, 1994, p. 4). Aunque este término ha sido conceptualizado operacionalmente como un rasgo de personalidad, una habilidad, o una cualidad 
de la atención, diversos autores apuntan hacia una definición en términos de conductas particulares, manifestaciones de la experiencia y procesos cognitivos del individuo (Bishop et al., 2004), los cuales permanecen inherentes a la persona, y pueden ser entrenados para mejorar la salud mental y física (Keng, Smoskiy y Robins, 2011). En este sentido, el mindfulness puede encuadrarse como un rasgo disposicional del individuo, que puede perfeccionarse por medio de protocolos de entrenamiento, como el programa de reducción del estrés basado en mindfulness (MBSR, por sus siglas en inglés), y los programas de intervención terapéuticos en psicología, basados en mindfulness (Baer, 2003; Creswell, 2017; Korman y Garay, 2012; Murphy, 2016).

Los mecanismos de acción del mindfulness, han sido teorizados como procesos cognitivos subyacentes que se traslapan con la regulación psicológica, y cuyos elementos fungen como moduladores de conductas con relación a la mejora de la salud mental y prevención de sintomatología clínica, por ejemplo: la atención enfocada al momento presente; la actitud de aceptación de la experiencia directa; la comprensión de las experiencias internas del individuo; y la regulación emocional (Coffey, Hartman y Fredrickson, 2010; Hayes y Feldman, 2004). Bajo este paradigma, se ha demostrado que el mindfulness disposicional y entrenado puede ayudar a lidiar y proteger a un individuo contra una serie de dificultades psicológicas, cuyas dimensiones pueden ayudar a predecir el consumo problemático de sustancias psicoactivas, el bienestar subjetivo, la resiliencia, el estrés y la sintomatología depresiva y ansiosa en distintos grupos de edad (Brown y Ryan, 2003; Davis y Hayes, 2011; Desrosiers, Vine, Klemanski y Nolen-Hoeksema, 2013; Gander, Proyer, Ruch, y Wyss, 2013; Karyadi, VanderVeen y Cyders, 2014; Pepping, Duvenage, Cronin y Lyons, 2016).

Por otro lado, tanto la espiritualidad como la religión han cobrado fuerte presencia en la investigación psicológica y las ciencias de la salud (Cullen, 2016; Lucchetti y Lucchetti, 2014). Estos dos términos han sido controversiales con respecto a sus definiciones y medición, pero son difíciles de ignorar, en el sentido de que forman parte importante de un proceso histórico, social colectivo e individual de la humanidad, y que por lo tanto no pueden descartarse. Aunque la espiritualidad y la religión aparenten ser dos caras de un mismo fenómeno, algunos autores enfatizan en que ambos términos son entidades relacionadas, pero independientes (Saucier y Skrzypińska, 2006; Van Niekerk, 2018). Por lo tanto, debido al traslape conceptual y práctico entre estos términos, en este estudio consideramos conveniente categorizar ambos aspectos en los individuos como grupos religiosos/espirituales, de modo que se incluyan las afiliaciones seculares 
no explícitamente religiosas, como quienes dicen ser "espirituales, pero no religiosos". Adicionalmente, se ha sugerido que la espiritualidad y la religiosidad, sean consideradas como rasgos de personalidad asociados a la resiliencia, humildad, honestidad y altruismo (Aghababaei, Wasserman y Nannini, 2014; MacDonald, 2000; Rodríguez, Fernández, Pérez y Noriega, 2011; Salgado, 2014; Womble, Labbé y Cochran, 2013).

Para propósitos del estudio, se consideró la definición de religión propuesta por Koenig (2011) quien la describe como un "sistema organizado de creencias, prácticas y símbolos diseñados para facilitar la cercanía a lo trascendente o lo Divino y fomentar la comprensión de la relación y las responsabilidades de uno con los demás que viven en comunidad" (p. 194), mientras que para la espiritualidad, se usó la definición proporcionada por la Conferencia del Consenso Internacional en 2012, que estipula que la espiritualidad es:

un aspecto dinámico e intrínseco de la humanidad a través del cual las personas buscan el significado, el propósito y la trascendencia fundamentales, y experimentan una relación con uno mismo, la familia, los demás, la comunidad, la sociedad, la naturaleza y lo significativo o lo sagrado. La espiritualidad se expresa a través de creencias, valores, tradiciones y prácticas (Puchalski, Vitillo, Hull y Reller, 2014, p. 646).

Una perspectiva importante en el estudio científico de la religión y la espiritualidad, reside principalmente en los procesos psicológicos y contextuales funcionales relacionados con el significado individual y colectivo, y con las contingencias resultantes de los comportamientos asociados a estos (Hayes, 1984; Martin y George, 2016), similarmente, la importancia de estudiar estos fenómenos se encuentra en los efectos que éstas poseen sobre las personas de manera integral y en sus diferentes áreas de funcionamiento, independientemente de su grupo de edad. Por ejemplo, existen estudios que, considerando uno o ambos términos, han puesto de manifiesto los papeles protectores en la salud mental (Barbosa Felipe, Pimenta Carvalho y Baptista Andrade, 2015; Cotton, Zebracki, Rosenthal, Tsevat y Drotar, 2006; Koenig, 2012; Vargas-Valle y MartínezCanizales, 2015).

En la adolescencia, los individuos son particularmente vulnerables a conductas de riesgo, asimismo, esta etapa de la vida supone una cantidad considerable de estrés, puntualmente inducida por los cambios biológicos, cognitivos y sociales que normalmente se experimentan en esta etapa 
del desarrollo (Veytia López, González Arratia López Fuentes, Andrade Palos y Oudhof, 2012). Sin embargo, es también aquí donde el proceso de formación de identidad y consolidación de gustos y preferencias es fuerte y maleable, de manera que la adquisición de habilidades y significados personales, como las conductas relacionadas con el mindfulness y las prácticas espirituales, pueden impactar de manera positiva en el desarrollo de la personalidad y los estilos de vida. Considerando que desde el año 2011, en México el consumo de sustancias psicoactivas como el alcohol, el tabaco y las drogas ilegales ha ido en aumento en población adolescente (Comisión Nacional contra las Adicciones - CONADIC, 2017a, 2017b, 2017c), es prudente que la investigación en psicología de la salud integre nuevas perspectivas en la cultura de la prevención de las adicciones y promoción de la salud, tanto a nivel individual como comunitario; adicionalmente, la investigación en fenómenos como el mindfulness y la espiritualidad es muy limitada en México.

Así entonces que el objetivo principal de este estudio fue evaluar las relaciones entre mindfulness disposicional, espiritualidad y religión, y su papel como factores protectores del consumo de sustancias psicoactivas en adolescentes, además de analizar las diferencias en las puntuaciones de mindfulness y espiritualidad entre consumidores y no consumidores de sustancias, y entre los diferentes grupos religiosos/espirituales.

\section{Método}

\section{Muestra}

Mediante un método de muestreo no probabilístico por conveniencia, se consideraron inicialmente un total de 473 estudiantes adolescentes de una institución educativa de nivel medio superior de la ciudad de Toluca, Estado de México. Se incluyeron participantes de ambos sexos de entre 14 y 18 años. Se excluyeron aquellos participantes que actualmente estaban recibiendo tratamiento psicológico o psiquiátrico $(n=36)$ y los grupos religiosos/espirituales con escasa distribución $(n=$ 4); se contó al final con una muestra de $n=433$, con una media de edad de 16.28 años (DE \pm .91 ), compuesto por $62.2 \%$ de mujeres $(n=270)$ y $37.8 \%$ de varones $(n=163)$.

\section{Procedimiento}

El proyecto del presente estudio se sometió a evaluación del Comité de Bioética del Centro de Investigación en Ciencias Médicas (CICMED) de la Universidad Autónoma del Estado de México 
(UAEMEX). Se solicitó un permiso por parte del plantel académico con las autoridades para acordar horarios y grupos disponibles de aplicación. Previo a la aplicación de los instrumentos, se entregaron grupalmente cartas de asentimiento informado a los estudiantes, y se requirió además el consentimiento informado de su padre, madre o tutor explicando el propósito del proyecto y las consideraciones éticas. Una vez que ambos documentos fueron recogidos y revisados, se continuó con la aplicación de instrumentos en un plazo de aproximadamente treinta minutos por aplicación grupal. Los datos fueron capturados y analizados estadísticamente por computadora.

\section{Instrumentos}

Ficha Sociodemográfica. Formato donde se preguntaban datos generales, como sexo, el grupo religioso/espiritual, consumo de sustancias (alcohol, drogas y tabaco), situación laboral, orientación sexual y estado conyugal de los padres. La variable de grupos religiosos/espirituales a los que los sujetos se identificaban actualmente, se manejó con cinco categorías inicialmente: 1) Sin Religión; 2) Católico; 3) Cristiano; 4) Espiritual pero no Religioso; y 5) testigo de Jehová, considerando grupos nacionales reportados por la Encuesta Nacional sobre Creencias y Prácticas Religiosas (RIFREM, 2016). Sin embargo, debido a la escasa proporción de testigos de Jehová ( $n$ = 4; 2 mujeres), estos fueron eliminados posteriormente de los análisis. La variable de consumo de sustancias psicoactivas se manejó de manera dicotómica, donde se preguntó si actualmente consumían tabaco o alcohol, si habían consumido drogas ilegales, o si nunca habían consumido alguno de estos tipos de sustancias en absoluto. Las drogas ilegales se ejemplificaron como: marihuana/cannabis, cocaína, LSD, hongos alucinógenos, inhalantes, éxtasis/MDMA, y cristal/metanfetaminas.

Escala de Atención Consciente (MAAS). Este instrumento fue diseñado por Brown y Ryan (2003) y ha sido satisfactoriamente adaptado a la población mexicana (López-Maya et al., 2015). Es una escala de autoreporte compuesto de 15 ítems en escala Likert, con una puntuación mínima de 15 y máxima de 90, que tiene como objetivo la medición de las habilidades disposicionales de mindfulness en función de la frecuencia de expresión de conductas directamente relacionadas con el constructo con un factor único, donde la carga factorial explica $43.62 \%$ de la varianza total y se reporta un índice de confiabilidad de $\alpha=.89$ en población normativa; para este estudio, el nivel $\alpha$ fue $=.90$. 
Cuestionario de Espiritualidad (SQ). Desarrollado inicialmente por Parsian y Dunning (2009) y adaptado al idioma español (Díaz Heredia, Muñoz Sánchez y De Vargas, 2012), es un instrumento con 29 ítems en escala tipo Likert divididos en subescalas: 1) Autoconsciencia (AC); 2) Importancia de las Creencias Espirituales (ICE); 3) Prácticas Espirituales (PES); y 4) Necesidades Espirituales (NE), el cual pretende medir el grado de acuerdo con respecto a la búsqueda de sentido en la vida, autoactualización, y conexión con uno mismo y con el universo. La puntuación mínima es de 29, y la máxima de 116. La confiabilidad de este instrumento es de $\alpha=.88$, y los cuatro factores que le componen explican el $52.60 \%$ de la varianza; para este estudio, el nivel de $\alpha$ fue de .91 .

\section{Análisis estadístico}

Se realizó la prueba de bondad de ajuste de Kolmogorov-Smirnov para confirmar la distribución de normalidad en las variables, y se elaboró un análisis descriptivo de frecuencias y porcentajes. Se usaron pruebas de correlación producto-momento de Pearson y Spearman para identificar la magnitud de la relación entre las variables de Consumo de Sustancias Psicoactivas (Alcohol, Tabaco, Drogas Ilegales), Religión, Mindfulness y Espiritualidad y las subescalas de este instrumento. Se ejecutó la prueba $t$ de Student (la prueba $U$ de Mann-Whitney en el caso de varianzas desiguales) y la prueba de análisis de varianza (ANOVA) unidireccional para analizar las diferencias entre consumidores y no consumidores de sustancias, y entre los distintos grupos religiosos/espirituales con respecto a las puntuaciones en Mindfulness y Espiritualidad, respectivamente. Finalmente, se crearon modelos de regresión logística binaria para examinar la probabilidad de consumo de sustancias. Se asumió un nivel de confianza en las pruebas de $95 \%$. Los datos fueron procesados en el programa IBM SPSS v.23 ®.

\section{$\underline{\text { Resultados }}$}

La muestra estuvo conformada por 433 participantes, de los cuales $62.2 \%$ fueron mujeres $(n=$ 270) y $37.8 \%$ varones $(n=163)$. La media de edad para toda la muestra fue de 16.27 años (DE \pm $.91)$. Un $89.4 \%(n=387)$ de la muestra reportó ser heterosexual; $3.5 \%(n=15)$ mencionaron ser homosexuales; y $7.2 \%(n=31)$ bisexuales. Además, $14 \%$ de los participantes $(n=61)$ mencionaron estar involucrados actualmente en un trabajo remunerado; $20.1 \%(n=87)$ reportaron que sus padres se encontraban actualmente separados o divorciados; y $24.7 \%(n=107)$ tenían vida 
sexual activa. En la Tabla 1, se exponen las frecuencias y porcentajes del consumo de sustancias y de los grupos religiosos/espirituales, además de las puntuaciones de los instrumentos expresados en medias y desviaciones estándar. Con respecto a los grupos religiosos/espirituales, se observa que la mayoría de los participantes reportaron identificarse como Católicos $(n=303 ; 70 \%)$. Por otro lado, casi la mitad de la muestra reportó consumir alcohol actualmente (45.3\%), mientras que más de la mitad nunca había consumido tabaco (80.6\%), ni drogas ilegales $(83.8 \%)$.

Tabla 1. Consumo de sustancias, grupo religioso/espiritual y puntuaciones generales de los instrumentos.

Table 1. Substance use, religious/spiritual group, and general instrument scores.

\begin{tabular}{|lc|}
\hline & Total $(\boldsymbol{n}=\mathbf{4 3 3})$ \\
\hline Grupo Religioso/Espiritual & $\boldsymbol{n}(\boldsymbol{\%})$ \\
Espiritual, no Religioso (ENR) & $44(10.2)$ \\
Católico & $303(70)$ \\
Cristiano & $19(4.4)$ \\
Sin Religión (SR) & $67(15.5)$ \\
Consumo de Alcohol & \\
Actualmente consume & $196(45.3)$ \\
Nunca ha consumido & $237(54.7)$ \\
Consumo de Tabaco & \\
Actualmente Consume & $84(19.4)$ \\
Nunca ha consumido & $349(80.6)$ \\
Consumo de Sustancias Psicoactivas Ilegales & $70(16.2)$ \\
Ha consumido alguna vez & $363(83.8)$ \\
Nunca ha consumido & Media \pm DE \\
\hline & \\
\hline Mindfulness & $68.29 \pm 13.18$ \\
Puntuación Total (MAAS Global) & \\
Espiritualidad & $31.77 \pm 5.66$ \\
Subescala Autoconsciencia (AC) & $11.27 \pm 2.75$ \\
Subescala Importancia de las Creencias Espirituales (ICE) & $15.50 \pm 3.52$ \\
Subescala Prácticas Espirituales (PES) & $29.79 \pm 3.94$ \\
Subescala Necesidades Espirituales (NES) & $88.33 \pm 12.50$ \\
Puntuación Total (SQ Global) &
\end{tabular}

Fuente: Elaboración propia.

Source: Own.

La primera sección de la Tabla 2 muestra los coeficientes de correlación de Pearson para las puntuaciones globales de Mindfulness (MAAS) y Espiritualidad (SQ) junto con las subescalas de este último, hallándose asociaciones positivas muy significativas, pero en su mayoría débiles ( $\mathrm{r}<$ .5). En la segunda sección de esta misma tabla, se muestran los coeficientes de correlación de Spearman para las puntuaciones globales de estos dos instrumentos con el Consumo de Sustancias (tabaco, alcohol, drogas ilegales) y Religión. Para la evaluación de esta última, se creó una variable dicotómica artificial a partir de los Grupos Religiosos/Espirituales, en donde se agruparon a quienes 
no profesaban ninguna religión propiamente (SR, ENR) y a quienes sí (Católico, Cristiano) ( $n=$ 322). La puntuaciones en MAAS Global se correlacionaron negativamente y con una fuerza débil con el consumo de sustancias psicoactivas (alcohol, tabaco y drogas), y de manera positiva con el hecho de profesar una religión, estos resultados fueron estadísticamente significativos. Por otro lado, únicamente las puntuaciones en Espiritualidad se correlacionaron de manera positiva y con una fuerza débil con el hecho de profesar una religión $(\mathrm{p}<.05)$.

Tabla 2. Coeficientes de correlación de Pearson para Mindfulness (MAAS) y el Cuestionario de Espiritualidad (SQ) con subescalas.

Table 2. Pearson's correlation coefficients for Mindfulness (MAAS) and the Spirituality Questionnaire (SQ) with subscales.

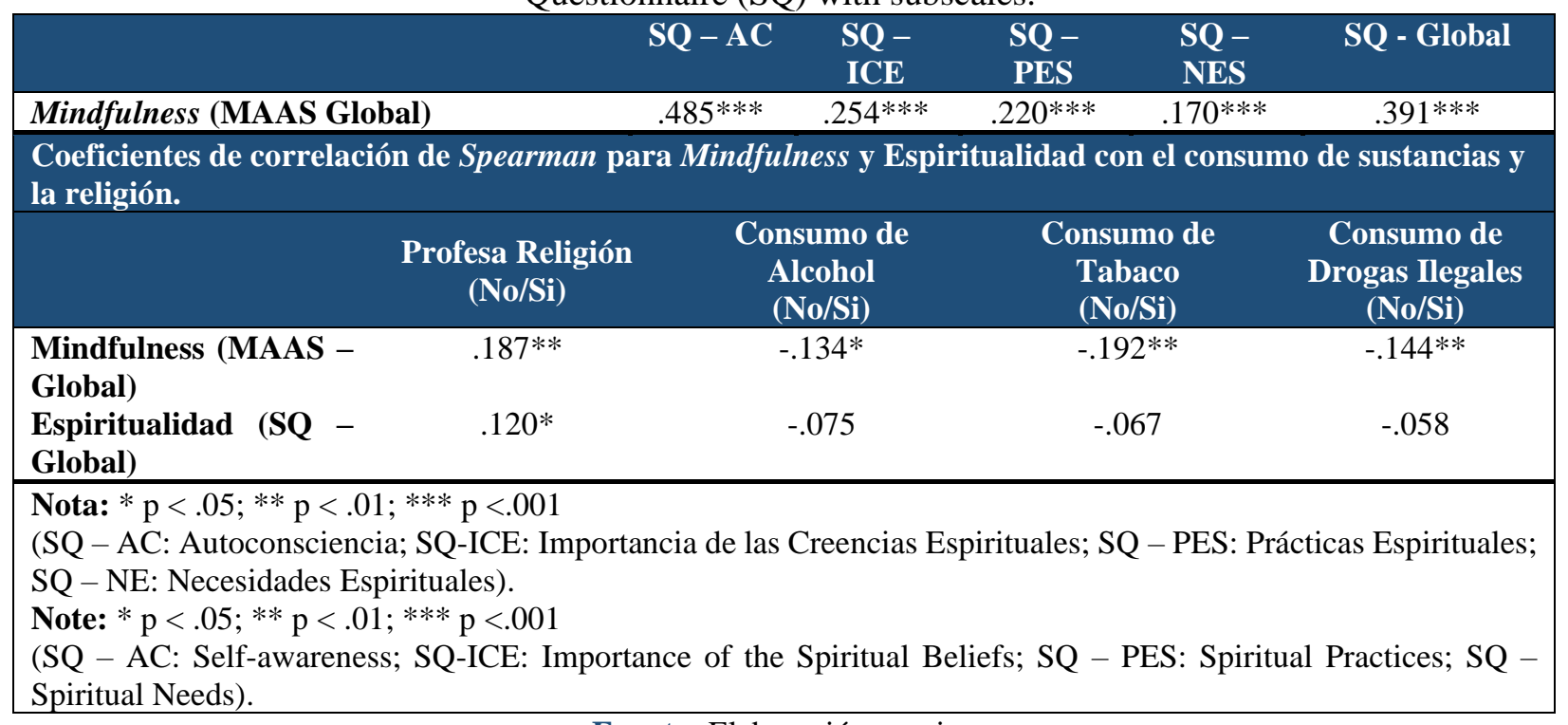

$$
\begin{aligned}
& \text { Fuente: Elaboración propia. } \\
& \text { Source: Own. }
\end{aligned}
$$

La variable dicotómica de Religión también se usó para contrastar los valores esperados de los observados con respecto al consumo de sustancias psicoactivas mediante la prueba de $\chi^{2} 2 \times 2$. Se encontraron asociaciones estadísticamente significativas entre el hecho de profesar una religión, con el consumo de tabaco $(\mathrm{p}<.001)$, alcohol $(\mathrm{p}<.01)$ y drogas ilegales $(\mathrm{p}<.001)$.

Al realizar la prueba de $t$ de Student $(\mathrm{IC}=95 \%$ ), se encontró que las puntuaciones medias en MAAS Global de quienes nunca habían consumido alcohol y tabaco, fueron significativamente mayores a quienes sí las consumían actualmente ( $\mathrm{p}<.001$, dos colas). Por otro lado, no existieron diferencias estadísticamente significativas con respecto al consumo de alcohol y tabaco en las puntuaciones de SQ Global, ni en ninguna de las subescalas del instrumento. Estos resultados se 
observan en la Tabla 3. Debido a que no se cumplió con el criterio de homocedasticidad para las categorías de consumo de drogas ilegales, para analizar las diferencias en estos grupos se usó la prueba $U$ de Mann-Whitney, exponiendo diferencias significativas en Mindfulness $(U=9834, p=$ .003 , bilateral) pero no en Espiritualidad $(p=.796)$ (no mostrado en tabla).

Tabla 3. Resultados de $t$ de Student para las diferencias en MAAS y SQ entre consumidores y no consumidores de sustancias psicoactivas.

Table 3. Student's t test results for the differences in MAAS and SQ between users and non-users of psychoactive substances.

\begin{tabular}{|c|c|c|c|c|c|c|}
\hline & \multicolumn{6}{|c|}{ Valores $t$} \\
\hline & $\begin{array}{c}\text { MAAS - } \\
\text { Global }\end{array}$ & $\begin{array}{c}\text { SQ - } \\
\text { Global }\end{array}$ & $\mathbf{S Q}-\mathbf{A C}$ & SQ-ICE & SQ - PES & $\mathbf{S Q}-\mathbf{N E}$ \\
\hline Consumo de Alcohol & $3.126^{*}$ & 1.188 & .677 & 1.971 & 1.737 & -.129 \\
\hline Consumo de Tabaco & $4.251 * *$ & 1.141 & 1.085 & 1.383 & 1.751 & -.470 \\
\hline \multicolumn{7}{|c|}{$\begin{array}{l}\text { Nota: } * \mathrm{p}<.01 ; * * \mathrm{p}<0.001 \\
\text { (SQ - AC: Autoconsciencia; SQ-ICE: Importancia de las Creencias Espirituales; SQ - PES: Prácticas Espirituales; } \\
\text { SQ - NE: Necesidades Espirituales) } \\
\text { Note: } * \mathrm{p}<.01 ; * * \mathrm{p}<0.001 \\
\text { (SQ - AC: Self-awareness; SQ-ICE: Importance of the Spiritual Beliefs; SQ - PES: Spiritual Practices; SQ - } \\
\text { Spiritual Needs) }\end{array}$} \\
\hline
\end{tabular}

Fuente: Elaboración propia.

Source: Own.

Para poder analizar las diferencias entre las puntuaciones de Mindfulness y Espiritualidad considerando las cuatro categorías de la variable Grupo Religioso/Espiritual, se llevó a cabo la prueba de análisis de varianza (ANOVA) unidireccional, los resultados de esta prueba pueden observarse en la Tabla 4, con excepción de la subescala SQ - ICE, que, de acuerdo con el estadístico de Levene, no cumplió con el criterio de homocedasticidad y no es presentada. 
Tabla 4. Análisis de varianza (ANOVA) para MAAS y SQ con el factor Grupo Religioso/Espiritual.

Table 4. Analysis of variance (ANOVA) for MAAS and SQ with Religious/Spiritual Group

as factor.

\begin{tabular}{|c|c|c|c|c|c|c|}
\hline & \multicolumn{4}{|c|}{ Media (DE) } & \multirow[b]{2}{*}{$\begin{array}{c}\text { Cociente } \\
\text { F }\end{array}$} & \multirow[b]{2}{*}{$\begin{array}{c}\text { Valor } \\
p\end{array}$} \\
\hline & $\begin{array}{l}\text { Sin } \\
\text { Religión } \\
\text { (SR) }\end{array}$ & Católico & Cristiano & $\begin{array}{c}\text { Espiritual No } \\
\text { Religioso } \\
\text { (ENR) }\end{array}$ & & \\
\hline \multicolumn{7}{|l|}{ Espiritualidad (SQ) } \\
\hline Subescala & 30.84 & 32.04 & 33.16 & 30.75 & 1.693 & .168 \\
\hline Autoconsciencia (AC) & $(5.71)$ & $(5.70)$ & $(4.42)$ & $(5.61)$ & & \\
\hline $\begin{array}{l}\text { Subescala Prácticas } \\
\text { Espirituales (PES) }\end{array}$ & $\begin{array}{l}14.70 \\
(3.59)\end{array}$ & $\begin{array}{l}15.76 \\
(3.49)\end{array}$ & $\begin{array}{c}14 \\
(3,30)\end{array}$ & $\begin{array}{l}15.52 \\
(3.51)\end{array}$ & 2.886 & 035 \\
\hline Subescala Necesidades & 28.97 & 29.92 & 29.16 & 30.43 & 1637 & \\
\hline Espirituales (NE) & $(4.55)$ & $(3.86)$ & (3) & $(3.80)$ & 1.037 & . \\
\hline Puntuación Total (SQ & 84.27 & $\begin{array}{l}89.36 \\
(1220)\end{array}$ & 87.84 & 87.57 & 3.157 & .025 \\
\hline Mindfulness (MAAS) & & & & & & \\
\hline $\begin{array}{l}\text { Puntuación Total } \\
\text { (MAAS Global) }\end{array}$ & (11.26) & $\begin{array}{l}09.45 \\
(12.88)\end{array}$ & (14.19) & $\begin{array}{l}01.25 \\
(15.04)\end{array}$ & 6.438 & .001 \\
\hline
\end{tabular}

Fuente: Elaboración propia.

Source: Own.

Se observaron diferencias estadísticamente significativas con respecto a las cuatro categorías de la variable Grupo Religioso/Espiritual en las puntaciones de Mindfulness (MAAS Global) $[F(3,429)$ $=6.438, p=.001]$, Espiritualidad (SQ Global) $[F(3,429)=3.157, p=.025]$ y la subescala de Prácticas Espirituales $(\mathrm{PES})[F(3,429)=2.886, p=.035]$ de este instrumento. Los contrastes posthoc con la prueba $t$ de Bonferroni mostraron que las puntuaciones medias en MAAS Global fueron significativamente mayores en los grupos de Cristiano y Católico que en los $E N R$ (p <.01), pero no en $S R(p>.05)$, mientras que las puntuaciones medias en SQ Global fueron significativamente mayores en el grupo de Católico en comparación con SR ( $<$.05).

Finalmente, se crearon modelos de regresión logística binaria con intervalos de confianza del $95 \%$ para evaluar la probabilidad del consumo de sustancias a partir de las variables predictoras Religión (dicotómica artificial), Mindfulness (MAAS Global) y Espiritualidad (SQ Global). Sin embargo, esta última variable se eliminó de los modelos debido a que no contribuía significativamente a ninguno de ellos $(\mathrm{p}>.05)$. De acuerdo con la prueba de Hosmer y Lemeshow, no fue adecuado el modelo de predicción para el consumo de tabaco $\left[\chi^{2}(8, N=433)=18.810, p\right.$ $=.016$ ] por lo que no se presenta en la tabla. El ajuste de los dos modelos restantes fue bajo, y con una sustancial cantidad de varianza no explicada (para Alcohol: $-2 L L=580.221, R^{2}$ de Cox y Snell $=.037, R^{2}$ de Nagelkerke $=.049$; para Drogas: $-2 L L=353.765, R^{2}$ de Cox y Snell $=.066, R^{2}$ de 
Nagelkerke $=.112$ ). De acuerdo con el modelo de predicción de Consumo de Alcohol, la pertenencia a la religión fue la principal variable predictora de consumo $(\operatorname{Exp}(B)=.561)$ actuando de manera inversa, lo que significa que los individuos que pertenecían formalmente una religión (en este caso Católico o Cristiano) reducían la probabilidad de haber consumido alcohol 1/.561= 1.7 veces (OR) a comparación de quienes no pertenecían a una (SR, ENR). Con respecto al Consumo de Drogas Ilegales, la pertenencia a una religión también actuaba inversamente, reduciendo la probabilidad de consumo con una razón de aproximadamente 3 veces a $1(\operatorname{Exp}(B)=$ $.323 ; \mathrm{OR}=1 / .323=3.09$ ). Con respecto a la variable de mindfulness, la probabilidad fue casi nula en ambos modelos (para Consumo de Alcohol: $\operatorname{Exp}(B)=.908$; para Consumo de Drogas Ilegales: $\operatorname{Exp}(B)=.971)$, aunque estadísticamente significativa, actuando también de manera inversa. La información completa de los modelos es presentada en la Tabla 5.

Tabla 5. Modelos de regresión logística binaria para consumo de sustancias (alcohol y drogas ilegales).

Table 5. Binary logistic regression models for substance use (alcohol and illegal drugs).

\begin{tabular}{|c|c|c|c|c|c|c|c|c|}
\hline & & B & $\begin{array}{c}\text { Error } \\
\text { Estándar }\end{array}$ & $\chi^{2}$ Wald & Valor $p$ & $\begin{array}{c}\operatorname{Exp}(\mathbf{B}) \\
(\mathrm{OR})\end{array}$ & $\begin{array}{c}\text { IC } \\
\text { Inferior }\end{array}$ & $\begin{array}{c}\text { IC } \\
\text { Superior }\end{array}$ \\
\hline Paso 1. & $\begin{array}{l}\text { Religión } \\
\text { Mindfulness } \\
\text { (MAAS } \\
\text { Global) }\end{array}$ & $\begin{array}{l}-.578 \\
-.020\end{array}$ & .227 & $\begin{array}{l}6.459 \\
6.818\end{array}$ & .011 & .561 & .360 & .876 \\
\hline \multicolumn{9}{|c|}{ Modelo 2. Consumo de Drogas Ilegales. } \\
\hline Paso 1. & $\begin{array}{l}\text { Religión } \\
\text { Mindfulness } \\
\text { (MAAS } \\
\text { Global) }\end{array}$ & $\begin{array}{r}-1.129 \\
-.030\end{array}$ & $\begin{array}{l}.277 \\
.010\end{array}$ & $\begin{array}{l}16.645 \\
8.622\end{array}$ & .001 & .323 & $\begin{array}{l}.188 \\
.952\end{array}$ & .556 \\
\hline
\end{tabular}

Fuente: Elaboración propia.

Source: Own.

\section{Discusión}

El objetivo general de este estudio fue evaluar las relaciones entre mindfulness disposicional, espiritualidad y religión, y su papel como factores protectores del consumo de sustancias psicoactivas en adolescentes, además de analizar las diferencias en las puntuaciones de mindfulness y espiritualidad entre consumidores y no consumidores de sustancias, y entre los diferentes grupos religiosos/espirituales.

A través de un análisis descriptivo se pudo observar que las puntuaciones medias de los instrumentos de mindfulness (MAAS Total) y espiritualidad (SQ Total) para ambos sexos se 
reportaron por encima de la mediana de la puntación global de ambos instrumentos, lo que puede ser explicado debido a que fueron aplicados en población no clínica, en donde podría esperarse esta tendencia, por lo menos en la variable de mindfulness (Tan y Martin, 2012). Otros estudios parecen sugerir inversamente esta tendencia, en donde se han encontrado puntuaciones más bajas de mindfulness disposicional en población que posee síntomas de depresión, ansiedad, o reactividad cognitiva y dificultades para regular las emociones (Creswell, 2017; Pepping et al., 2016).

Con relación al consumo de alcohol, casi la mitad del total de la muestra (45.3 \%) reportó consumirlo actualmente, datos que no difieren mucho con respecto a lo reportado previamente en la última encuesta nacional mexicana (CONADIC, 2017a), donde se expuso una prevalencia de consumo de "alguna vez" en un 39.8 \% para ambos sexos en población adolescente de 12 a 17 años. En contraste, los datos reportados para el consumo de tabaco y drogas ilegales son mucho más elevados en el presente estudio, en comparación con los reportados en las encuestas nacionales (CONADIC, 2017b, 2017c) para adolescentes, ya que la prevalencia de fumadores es de $4.9 \%$ y la obtenida en este estudio es de $19.4 \%$, mientras que en drogas ilegales es de $6.4 \%$ para cualquier droga, y la presente muestra reportó un $16.2 \%$.

Al examinar las relaciones entre las variables de mindfulness y espiritualidad, los coeficientes de correlación fueron positivos, y estadísticamente significativos, con una fuerza de leve a moderada. Estas tendencias son similares a las reportadas previamente, por ejemplo Carmody y sus colaboradores (2008) hallaron una relación predictiva positiva entre mindfulness y bienestar espiritual $(\beta=0.40, p=.001)$, y Lazaridou y Pentaris (2016) reportaron correlaciones positivas entre Mindfulness, Orientación Cognitiva hacia la Espiritualidad, y Espiritualidad experiencial ( $r=.41, r=.37$, respectivamente); las relaciones entre estas variables podrían estar influenciadas por la subescala de Autoconsciencia del SQ, ya que de acuerdo con Coffey, Hartman, y Fredrickson (2010) al menos dos mecanismos de acción fundamentales del mindfulness son la atención al presente y la comprensión de las experiencias internas, lo que ultimadamente involucra la consciencia del sí-mismo (Harrington, Loffredo y Perz, 2014), y los cambios en los estímulos experimentados momento a momento en el individuo.

Se encontró una relación débil, pero estadísticamente significativa entre la profesión de religión y la espiritualidad, que podría deberse al traslape teórico entre ambos conceptos en la investigación (Koenig, 2012), por otro lado, se encontraron diferencias significativas entre la 
pertenencia a la religión y el consumo de sustancias, mientras que las relaciones entre la espiritualidad y el consumo de sustancias no fueron significativas, ni considerablemente fuertes en magnitud. Adicionalmente, tampoco se encontraron diferencias significativas entre los grupos de consumidores y no consumidores con respecto a las puntuaciones en la variable de espiritualidad. Contrastar estos resultados con investigaciones previas resulta controversial, dado que se han reportado ambas variables como factores protectores ante el consumo de sustancias en poblaciones parecidas (Barbosa Felipe et al., 2015; Hodge, Cárdenas y Montoya, 2001; Miller, Davies y Greenwald, 2000; Vargas-Valle y Martínez-Canizales, 2015), sin embargo, estos resultados apuntan la tendencia sólo hacia la religión, y sugieren que la espiritualidad podría ser independiente del consumo.

En el caso de la espiritualidad como factor de protección, su efecto sería explicado en función del significado subjetivo y dinámico del individuo (trascendencia), y no como una habilidad disposicional (Carey, 2018; Martin y George, 2016; Van Niekerk, 2018). Es importante mencionar que la espiritualidad ha sido asociada a la resiliencia (Rodríguez et al., 2011; Salgado, 2014), de modo que podría ser más acertado considerar a la espiritualidad como un estilo de afrontamiento, antes que como un factor protector.

Las medias y rangos de las puntuaciones de mindfulness disposicional fueron significativamente mayores en aquellos individuos que no habían consumido ninguna sustancia en comparación con los que sí lo habían hecho o continuaban haciéndolo, además, para toda la muestra se encontró una ligera correlación inversa y significativa entre el mindfulness disposicional y el consumo de sustancias (alcohol, tabaco y drogas ilegales). Las relaciones entre mindfulness disposicional y consumo de sustancias han sido teorizadas con respecto a la orientación de la atención hacia la experiencias sensoriales y las alteraciones cognitivas, como resultado de consumir una sustancia psicoactiva, esto es, la funcionalidad de las conductas relacionadas con el mindfulness dependiendo de la experiencia. Por ejemplo, un individuo puede centrarse con mayor o menor frecuencia en el estado sensorial y cognitivo de una sustancia con el objetivo de acrecentar las experiencias y emociones positivas (por ejemplo, con el consumo de alcohol consuetudinario) o de reducir el impacto de las emociones negativas (consumo de tabaco y drogas como método de afrontamiento o evitación de lo aversivo) (Leigh, Bowen y Marlatt, 2005).

Los resultados del análisis de varianza (ANOVA) y la prueba $t$ de Bonferroni, parecen sugerir una tendencia a mayor mindfulness disposicional considerando la pertenencia formal a una 
religión, ya que las diferencias por grupo religioso/espiritual son puestas de relieve por el grupo de Espirituales No Religiosos y los No Religiosos, a comparación de los Católicos y Cristianos. Considerando estas diferencias, las implicaciones sociales y sanitarias de la espiritualidad secular han sido debatidas recientemente por King et al. (2013) en Estados Unidos y Vittengl (2018) en el Reino Unido, donde estos estudios controversialmente parecen indicar un riesgo a una peor salud mental por quienes se consideran Espirituales No Religiosos, pese a la creciente evidencia que apunta a la espiritualidad como un factor protector y resiliencia para la salud mental.

Finalmente, los modelos de regresión logística para el consumo de sustancias tuvieron un ajuste bajo. Es posible que un análisis más estricto de las variables predictoras pueda solidificar los resultados. No obstante, es importante considerar que la religión fue la principal variable predictora del consumo de alcohol y drogas ilegales, seguida por el mindfulness disposicional, ambas actuando inversamente. De acuerdo con Marsiglia, Kulis, Nieri y Parsai (2005) la religión fungiría como factor de protección con respecto al consumo de sustancias a través de normas culturales con base en un sistema de creencias morales establecido y relativamente rígido, el cual establece reglas particulares con respecto al consumo de sustancias, a comparación de la espiritualidad secular. Esta perspectiva podría explicar también la eliminación de la variable de espiritualidad en los modelos. Con respecto a la variable de mindfulness disposicional, de acuerdo con (Karyadi, VanderVeen y Cyders (2014), las interacciones dependen de las características de la población y su estado clínico, el tipo de sustancia consumida, y la severidad de su consumo, explicando que el mindfulness disposicional podría no ser una variable lo suficientemente robusta. Los presentes resultados parecen ser coherentes con lo sugerido.

En el presente estudio, se ha conceptualizado el mindfulness como un atributo disposicional, y que como tal puede entrenarse para incrementar su frecuencia en el comportamiento y la personalidad de manera que el individuo se beneficie (Baer, 2003); este grupo de investigación cree que la perspectiva del mindfulness como factor protector del consumo de sustancias psicoactivas, depende de los detonantes que podrían desencadenar el consumo problemático de sustancias psicoactivas, como el estrés (Bodenlos, Noonan y Wells, 2013; Ciesla, Reilly, Dickson, Emanuel y Updegraff, 2012), la baja regulación emocional, o los síntomas de depresión o ansiedad (Pepping et al., 2016); los cuales son particularmente más frecuentes en adolescentes debido a los factores contextuales de este grupo etario, incluyendo el ámbito académico, familiar e interpersonal (Veytia López et al., 2012). 


\section{Conclusiones}

Aunque las relaciones encontradas entre el consumo de sustancias y la religión son coherentes con lo anteriormente reportado en la literatura, aquellas encontradas entre el mindfulness y la espiritualidad con el consumo de sustancias parecen ser controvertidas.

Aunque el mindfulness no resultó como un predictor determinante del consumo de sustancias en nuestra muestra, existieron diferencias evidentes en esta variable entre quienes consumían sustancias psicoactivas y quienes no, y entre quienes profesaban formalmente una religión y quienes no. Con base en la literatura, el entrenamiento en mindfulness es útil en el tratamiento de los desencadenantes del consumo problemático de sustancias como la baja regulación emocional, los síntomas de ansiedad, depresión y estrés. En contraste, la espiritualidad secular al no ser una variable lo suficientemente robusta como para predecir el consumo de sustancias, podría fungir más apropiadamente como un estilo de afrontamiento, antes que un factor protector, en comparación con la religión.

La espiritualidad como la religión, al ser parte importante de los individuos, debe ser analizada cuidadosa y detalladamente en términos funcionales, de manera que se pueda complementar el conocimiento empírico, y analizar sus implicaciones sociales y sanitarias.

Los hallazgos obtenido en este estudio son novedosos en la población mexicana, sin embargo, sugerimos que futuras investigaciones deban asegurar un control metodológico más riguroso de las variables confusoras, tanto cualitativa como cuantitativamente, y una muestra más numerosa en orden de replicar o precisar los resultados presentados en este estudio.

\section{$\underline{\text { Agradecimientos }}$}

Los autores agradecen a las institución educativa que permitió la aplicación de los instrumentos, así como a las autoridades del plantel, a los alumnos de dicho plantel por su valiosa ayuda, al Consejo Nacional de Ciencia y Tecnología (CONACyT) por su soporte económico, y a la Universidad Autónoma del Estado de México (UAEMEX) por el respaldo y apoyo institucional. Los autores declaran no tener ningún conflicto de interés. 


\section{$\underline{\text { Referencias }}$}

Aghababaei, N., Wasserman, J. A. \& Nannini, D. (2014). The religious person revisited: crosscultural evidence from the HEXACO model of personality structure. Mental Health, Religion and Culture, 17(1), 24-29. DOI: https://doi.org/10.1080/13674676.2012.737771

Baer, R. A. (2003). Mindfulness training as a clinical intervention: A conceptual and empirical review. Clinical Psychology: Science and Practice, 10(2), 125-143. DOI: https://doi.org/10.1093/clipsy/bpg015

Barbosa Felipe, A. O., Pimenta Carvalho, A. M. \& Baptista Andrade, C. U. (2015). Spirituality and religion as protectors for adolescent drug use. SMAD. Revista Eletrônica Saúde Mental Álcool e Drogas, 11(1), 49-58. DOI: https://doi.org/10.11606/issn.1806-6976.v11i1p49-58

Bishop, S. R., Lau, M., Shapiro, S., Carlson, L., Anderson, N. D., Carmody, J., Segal, Z. V., Abbey, S., Speca, M., Velting D. \& Devins G. (2004). Mindfulness: A proposed operational definition. Clinical Psychology: Science and Practice, 11(3), 230-241. DOI: https://doi.org/10.1093/clipsy/bph077

Bodenlos, J. S., Noonan, M. \& Wells, S. Y. (2013). Mindfulness and alcohol problems in college students: The mediating effects of stress. Journal of American College Health, 61(6), 371378. DOI: https://doi.org/10.1080/07448481.2013.805714

Brown, K. W. \& Ryan, R. M. (2003). The Benefits of Being Present: Mindfulness and Its Role in Psychological Well-Being. Journal of Personality and Social Psychology, 84(4), 822-848. DOI: https://doi.org/10.1037/0022-3514.84.4.822

Carey, J. (2018). Spiritual, but not religious? On the nature of spirituality and its relation to religion. International Journal for Philosophy of Religion, 83(3), 261-269. DOI: https://doi.org/10.1007/s11153-017-9648-8

Carmody, J., Reed, G., Kristeller, J. \& Merriam, P. (2008). Mindfulness, spirituality, and healthrelated symptoms. Journal of Psychosomatic Research, 64(4), 393-403. DOI: https://doi.org/10.1016/j.jpsychores.2007.06.015

Ciesla, J. A., Reilly, L. C., Dickson, K. S., Emanuel, A. S. \& Updegraff, J. A. (2012). Dispositional Mindfulness Moderates the Effects of Stress Among Adolescents: Rumination as a Mediator. Journal of Clinical Child and Adolescent Psychology, 41(6), 760-770. DOI: https://doi.org/10.1080/15374416.2012.698724 
Coffey, K. A., Hartman, M. \& Fredrickson, B. L. (2010). Deconstructing Mindfulness and Constructing Mental Health: Understanding Mindfulness and its Mechanisms of Action. Mindfulness, 1(4), 235-253. DOI: https://doi.org/10.1007/s12671-010-0033-2

Comisión Nacional contra las Adicciones - CONADIC. (2017a). Encuesta Nacional de Consumo de Drogas, Alcohol y Tabaco 2016-2017: Reporte de Alcohol. Ciudad de México. Recuperado de de https://encuestas.insp.mx/ena/encodat2017/reporte_encodat_alcohol_2016_2017.pdf (23 de diciembre de 2019)

Comisión Nacional contra las Adicciones - CONADIC. (2017b). Encuesta Nacional de Consumo de Drogas, Alcohol y Tabaco 2016-2017: Reporte de Drogas. Ciudad de México. Recuperado de https://encuestas.insp.mx/ena/encodat2017/reporte_encodat_drogas_2016_2017.pdf(23 de diciembre de 2019)

Comisión Nacional contra las Adicciones - CONADIC. (2017c). Encuesta Nacional de Consumo de Drogas, Alcohol y Tabaco 2016-2017: Reporte de Tabaco. Ciudad de México. Recuperado de https://encuestas.insp.mx/ena/encodat2017/reporte_encodat_tabaco_2016_2017.pdf (23 de diciembre de 2019)

Cotton, S., Zebracki, K., Rosenthal, S. L. Tsevat, J., \& Drotar, D. (2006). Religion/spirituality and adolescent health outcomes: A review. Journal of Adolescent Health, 38(4), 472-480. DOI: https://doi.org/10.1016/j.jadohealth.2005.10.005

Creswell, J. D. (2017). Mindfulness Interventions. Annual Reviews of Psychology, 2017(68), 491516. DOI: https://doi.org/10.1146/annurev-psych-042716-051139

Cullen, J. G. (2016). Nursing management, religion, and spirituality: A bibliometric review, a research agenda, and implications for practice. Journal of Nursing Management, 24(3), 291-299. DOI: https://doi.org/10.1111/jonm.12340

Davis, D. M. \& Hayes, J. A. (2011). What Are the Benefits of Mindfulness? A Practice Review of Psychotherapy-Related Research. Psychotherapy, 48(2), 198-208. DOI: https://doi.org/10.1037/a0022062 
Desrosiers, A., Vine, V., Klemanski, D. H. \& Nolen-Hoeksema, S. (2013). Mindfulness and emotion regulation in depression and anxiety: Common and distinct mechanisms of action. Depression and Anxiety, 30(7), 654-661. DOI: https://doi.org/10.1002/da.22124

Díaz Heredia, L. P., Muñoz Sánchez, A. I. \& de Vargas, D. (2012). Confiabilidad y validez del cuestionario de espiritualidad de Parsian y Dunning en versión española. Rev. Latino-Am. Enfermagem, 20(3), 8.

Gander, F., Proyer, R. T., Ruch, W. \& Wyss, T. (2013). Strength-Based Positive Interventions: Further Evidence for Their Potential in Enhancing Well-Being and Alleviating Depression. Journal of Happiness Studies, 14(4), 1241-1259. DOI: https://doi.org/10.1007/s10902$\underline{012-9380-0}$

Harrington, R., Loffredo, D. A. \& Perz, C. A. (2014). Dispositional mindfulness as a positive predictor of psychological well-being and the role of the private self-consciousness insight factor. Personality and Individual Differences, 71, 15-18. DOI: https://doi.org/10.1016/j.paid.2014.06.050

Hayes, A. M. \& Feldman, G. (2004). Clarifying the construct of mindfulness in the context of emotion regulation and the process of change in therapy. Clinical Psychology: Science and Practice, 11(3), 255-262. DOI: https://doi.org/10.1093/clipsy/bph080

Hayes, S. C. (1984). Making sense of spirituality. Behaviorism, 12(2), 99-110. Recuperado de http://psycnet.apa.org/record/1986-05189-001 (4 de enero de 2020)

Hodge, D. R., Cárdenas, P. \& Montoya, H. (2001). Substance use: Spirituality and factors among rural youths. Social Work Research, 25(3), 153-161.

Kabat-Zinn, J. (1994). Wherever You Go, There You Are: Mindfulness Meditation in Everyday Life. New York: Hyperion.

Karyadi, K. A., VanderVeen, J. D. \& Cyders, M. A. (2014). A meta-analysis of the relationship between trait mindfulness and substance use behaviors. Drug and Alcohol Dependence, 143(1), 1-10. DOI: https://doi.org/10.1016/j.drugalcdep.2014.07.014

Keng, S.-L., J. Smoski, M. \& Robins, C. J. (2011). Effects of Mindfulness on Psychological Health: A Review of Empirical Studies. Clinical Psychology Review, 31(6), 1041-1056. DOI: https://doi.org/10.1016/j.cpr.2011.04.006. 
King, M., Marston, L., McManus, S., Brugha, T., Meltzer, H. \& Bebbington, P. (2013). Religion, spirituality, and mental health: Results from a national study of English households. British Journal of Psychiatry, 202(1), 68-73. DOI: https://doi.org/10.1192/bjp.bp.112.112003

Koenig, H. G. (2011). Spirituality \& Health Research: Methods, Measurements, Statistics, and Resources. West Conshohocken: Templeton Press.

Koenig, H. G. (2012). Religion, Spirituality, and Health: The Research and Clinical Implications. ISRN Psychiatry, 2012, 1-33. DOI: https://doi.org/10.5402/2012/278730

Korman, G. P. \& Garay, C. J. (2012). El modelo de Terapia Cognitiva basada en la conciencia plena (mindfulness). Revista Argentina de Clínica Psicológica, 21(1), 5-13

Lazaridou, A. \& Pentaris, P. (2016). Mindfulness and spirituality: therapeutic perspectives. PersonCentered and Experiential Psychotherapies, 15(3), 235-244. DOI: https://doi.org/10.1080/14779757.2016.1180634

Leigh, J., Bowen, S. \& Marlatt, G. A. (2005). Spirituality, mindfulness, and substance abuse. Addictive Behaviors, 30(7), 1335-1341. DOI: https://doi.org/10.1016/j.addbeh.2005.01.010

López-Maya, E., del Rocío Hernández-Pozo, M., Méndez-Segundo, L., Gutiérrez-García, J. J., Araujo-Díaz, D., Núñez-Gazcón, A., Cervantes-Sampayo, L. K., Nava-Alcántara, S., Bautista-García L. E. \& Hölzel B. K. (2015). Psychometric properties of the Mexican version of the Mindful Attention Awareness Scale (MAAS). Psychologia: Avances de la Disciplina, 9(1), 13-27.

Lucchetti, G. \& Lucchetti, A. L. G. (2014). Spirituality, Religion, and Health: Over the Last 15 Years of Field Research (1999-2013). The International Journal of Psychiatry in Medicine, 48(3), 199-215. DOI: https://doi.org/10.2190/PM.48.3.e

MacDonald, D. A. (2000). Spirituality: Description, Measurement, and Relation to the Five Factor Model of Personality. Journal of Personality, 68(1), 153-197. DOI: https://doi.org/10.1111/1467-6494.t01-1-00094

Marsiglia, F. F., Kulis, S., Nieri, T. \& Parsai, M. (2005). God Forbid! Substance Use Among Religious and Nonreligious Youth. The American journal of orthopsychiatry, 75(4), 585598. DOI: https://doi.org/10.1038/jid.2014.371

Martin, J. \& George, R. (2016). What is the point of spirituality? Palliative Medicine, 30(4), 325326. DOI: $\underline{\text { https://doi.org/10.1177/0269216316631931 }}$ 
Miller, L., Davies, M. \& Greenwald, S. (2000). Religiosity and substance use and abuse among adolescents in the national comorbidity survey. Journal of the American Academy of Child and Adolescent Psychiatry, 39(9), 1190-1197. DOI: https://doi.org/10.1097/00004583$\underline{200009000-00020}$

Murphy, A. (2016). Mindfulness-based Therapy in Modern Psychology: Convergence and Divergence from Early Buddhist Thought. Contemporary Buddhism, 17(2), 275-325. DOI: https://doi.org/https://doi.org/10.1080/14639947.2016.1228324

Parsian, N. \& Dunning, T. (2009). Developing and Validating a Questionnaire to Measure Spirituality: A Psychometric Process. Global Journal of Health Science, 1(1), 2-11. DOI: https://doi.org/10.5539/gjhs.v1n1p2

Pepping, C. A., Duvenage, M., Cronin, T. J. \& Lyons, A. (2016). Adolescent mindfulness and psychopathology: The role of emotion regulation. Personality and Individual Differences, 99, 302-307. DOI: https://doi.org/10.1016/j.paid.2016.04.089

Puchalski, C. M., Vitillo, R., Hull, S. K. \& Reller, N. (2014). Improving the spiritual dimension of whole person care: Reaching national and international consensus. Journal of Palliative Medicine, 17(6), 642-656. DOI: https://doi.org/10.1089/jpm.2014.9427

Red de Investigadores del Fenómeno Religioso en México (RIFREM). (2016). Encuesta Nacional sobre Creencias y Prácticas Religiosas. Ciudad de México. Recuperado de https://www.colef.mx/evento/encuesta-nancional-sobre-creencias-y-practicas-religiosasen-mexico-rifrem-2016/ (25 de diciembre de 2019)

Rodríguez, M., Fernández, M. L., Pérez, M. L. \& Noriega, R. (2011). Espiritualidad variable asociada a la resiliencia. Cuadernos Hispanoamericanos de Psicología, 11(2), 24-49.

Salgado, A. C. (2014). Revisión de estudios empíricos sobre el impacto de la religión, religiosidad y espiritualidad como factores protectores. Revista de Psicología Educativa de la USIL, 2(1), 121-140. DOI: https://doi.org/10.20511/pyr2014.v2n1.55

Saucier, G. \& Skrzypińska, K. (2006). Spiritual but not religious? Evidence for two independent dispositions. Journal of Personality, 74(5), 1257-1292. DOI: https://doi.org/10.1111/j.1467-6494.2006.00409.x

Tabei, S. Z., Zarei, N. \& Joulaei, H. (2016). The impact of spirituality on health. Shiraz E Medical Journal, 17(6). DOI: https://doi.org/10.17795/semj39053 
Tan, L. B. G. \& Martin, G. (2012). Mind full or mindful: A report on mindfulness and psychological health in healthy adolescents. International Journal of Adolescence and Youth, 21(1), 64-74. DOI: https://doi.org/10.1080/02673843.2012.709174

Van Niekerk, B. (2018). Religion and spirituality: What are the fundamental differences? HTS Teologiese Studies / Theological Studies, 74(3), 1-11. DOI: https://doi.org/10.4102/hts.v74i3.4933

Vargas-Valle, E. D. \& Martínez-Canizales, G. (2015). La relación entre el abuso del alcohol y la religión en adolescentes mexicanos. Población y Salud en Mesoamérica, 12(2), 1-21. DOI: https://doi.org/http://dx.doi.org/10.15517/psm.v12i2.16783

Veytia López, M., González Arratia López Fuentes, N. I., Andrade Palos, P. \& Oudhof, H. (2012). Depresión en adolescentes: El papel de los sucesos vitales estresantes. Salud mental, 35(1), $37-43$.

Vittengl, J. R. (2018). A Lonely Search? Risk for Depression When Spirituality Exceeds Religiosity. Journal of Nervous and Mental Disease, 206(5), 386-389. DOI: https://doi.org/10.1097/NMD.0000000000000815

Womble, M. N., Labbé, E. E. \& Cochran, C. R. (2013). Spirituality and Personality: Understanding Their Relationship to Health Resilience. Psychological Reports, 112(3), 706-715. DOI: https://doi.org/10.2466/02.07.PR0.112.3.706-715

Zinnbauer, B. J., Pargament, K. I., Cole, B., Rye, M. S., Butter, E. M., Belavich, T. G., Hipp, K. M., Scott A. B. \& Kadar J. L. (1997). Religion and Spirituality: Unfuzzying the Fuzzy. Journal for the Scientific Study of Religion, 36(4), 549. DOI: https://doi.org/10.2307/1387689 